\title{
3D SIMULATION OF PHOTOELECTRON CLOUD
}

\author{
L. Wang, H. Fukuma, K. Ohmi and Y. Suetsugu \\ High Energy Accelerator Research Organization (KEK), Tsukuba, Ibaraki 305-0801, Japan
}

\begin{abstract}
A 3-dimensional particle simulation code is developed to study the photoelectron cloud in positron rings. The effects of the various magnetic fields in KEKB LER are presented. The distribution and evolution of the electron-cloud are simulated.
\end{abstract}

\section{INTRODUCTION}

A blow-up of the vertical beam size is observed in the KEKB positron ring (LER)[1] and it is one of the serious problems limiting the luminosity of KEKB. F. Zimmerman and K. Ohmi [2-3] explained the blow-up as a single-bunch instability of a positron bunch due to electron cloud generated by photoemission and secondary emission. The blow-up depends on the electron cloud density near the beam. C-Yoke magnet, which consists of two permanent magnets and a Cshaped iron yoke, was installed in the ring. The effect of C-Yoke magnet was not very clear in suppressing the blow-up for short bunch spacing case, which is required for the physics runs. Then solenoid with total length of $800 \mathrm{~m}$ was installed in the LER ring in Sep. 2000 in order to clear the photoelectron near the beam. It was effective on reducing vertical blow-up [4]. Additional $430 \mathrm{~m}$ solenoid was installed in Jan. 2001. A 3D simulation code is developed to study the effects of these various kinds of magnetic field on the photoelectron formation, distribution, space charge effect, multipacting, and so on.

\section{COMPUTER PROGRAM}

The positron bunch is longitudinally divided into a number of slices according to Gaussian distribution. Photoelectrons are emitted when positron slices pass through a beam pipe with length $L$, which is usually chosen as 1 or $2 \mathrm{~m}$. A photoelectron yield of 0.1 is assumed in simulation and $30 \%$ of the photoelectrons are produced by the reflective photons. The center of photoelectron energy distribution is $5 \mathrm{eV}$ with $\mathrm{rms}$ (root mean square) energy spread of $5 \mathrm{eV}$. In our simulation, the photoelectrons are represented by macro-particles, which move in 3-dimensional space under the force:

$$
\mathbf{F}_{e}=\mathbf{F}_{p}+\mathbf{F}_{\text {space }}+\mathbf{F}_{B}
$$

where $\mathbf{F}_{\mathbf{p}}$ is the force by positron beam which is given by the Bassetti Formula and $\mathbf{F}_{\text {space }}$ is the space charge force of the photoelectron. A PIC 3D space charge solver has been developed to study the space-charge force [5]. $\mathbf{F}_{\mathrm{B}}$ is the force by magnetic field on the photoelectron. As a preliminary study, the space charge and secondary emission are not included in this study. They will be included one step by one step.

The parameters used in the simulation are shown in table 1.

\begin{tabular}{lll}
\hline Variable & Symbol & Value \\
\hline Ring circumference & $C$ & $3016.26 \mathrm{~m}$ \\
RF bucket length & $s_{r f}$ & $0.589 \mathrm{~m}$ \\
Bunch spacing & $s_{b}$ & $4 \mathrm{RF}$ buckets \\
Beam line density & $n_{b}$ & $1.5 \times 10^{10} \mathrm{~m}^{-1}$ \\
Bunch population & $N$ & $3.3 \times 10^{10}$ \\
Average vertical betatron & $\beta_{y}$ & $10 \mathrm{~m}$ \\
function & & \\
Average horizontal betatron & $\beta_{x}$ & $10 \mathrm{~m}$ \\
function & & \\
Horizontal emittance & $\varepsilon_{x}$ & $1.8 \times 10^{-8} \mathrm{~m}$ \\
Vertical emittance & $\varepsilon_{y}$ & $3.6 \times 10^{-10} \mathrm{~m}$ \\
Betatron tune & $v_{x} / v_{y}$ & $45.52 / 44.09$ \\
Rms bunch length & $\sigma_{l}$ & $4 \mathrm{~mm}$ \\
Chamber diameter & $2 R$ & $100 \mathrm{~mm}$ \\
\hline
\end{tabular}

Table 1: Parameters assumed for the simulation

\section{ELECTRON -CLOUD IN VARIOUS MAGNETIC FIELD}

The build up and the distribution of electron cloud (ecloud) in various magnetic fields are discussed in this section.

\subsection{Field free region}

Figure 1 shows a typical simulation result for a passage of 100 bunches with a bunch spacing of 4 RF buckets and a 480 buckets gap in field free region. In fig. 1(a) the unit of time is bunch spacing, i.e. 7.86ns(4 RF buckets). The exponential decay time of the electron cloud at the gap is $35 \mathrm{~ns}$. The average volume density of the electron cloud at saturation level is $2.78 \times 10^{11} \mathrm{~m}^{-3}$. The volume density at the central region is $8.83 \times 10^{11} \mathrm{~m}$ 3. The central region is defined as a circle with radius of $10 \mathrm{~mm}$ at the pipe center. Most electron cloud is confined in the central region of the vertical direction by the positron slices as shown in figure 1(b). 


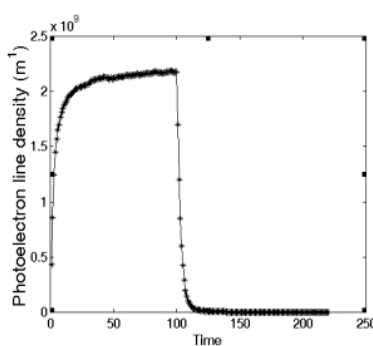

(a)

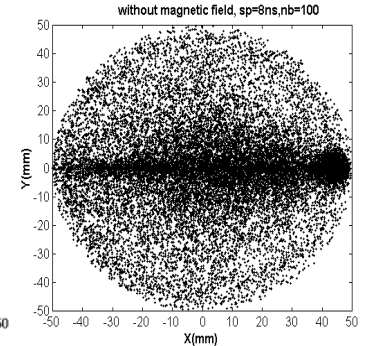

(b)
Figure $1 \quad$ Field Free Case

(a)Electron line density as a function of time

(b)Photoelectron distribution in transverse section. The is integrated longitudinally.

\subsection{C-Yoke magnet}

The C-Yoke magnet can be arranged in a dipole or quadrupole configuration. For the dipole arrangement of the $\mathrm{C}$ yoke magnet configuration, the field can be approximately expressed as

$$
\begin{aligned}
& B_{x}=0 \\
& B_{y}=a+b \cos (k z) \\
& B_{z}=-b k \sin (k z)
\end{aligned}
$$

where $a=141 \mathrm{G}, b=94 G, \lambda=0.1 \mathrm{~m}$ and $a=0, b=235 \mathrm{G}, \lambda=$ $0.2 \mathrm{~m}$ for the case of adjacent dipoles with equal polarity and alternating polarity, respectively. The distribution of photoelectrons in transverse section for these two fields is shown in figure 2. The average and central volume density are $1.07 \times 10^{11} \mathrm{~m}^{-3}$ and $0.75 \times 10^{11}$ $\mathrm{m}^{-3}$ for equal polarity case. They are $0.98 \times 10^{11} \mathrm{~m}^{-3}$ and $0.35 \times 10^{11} \mathrm{~m}^{-3}$ for alternating polarity case. In alternating polarity case, the volume density is lower and the saturation time is large compared with those in equal polarity case.

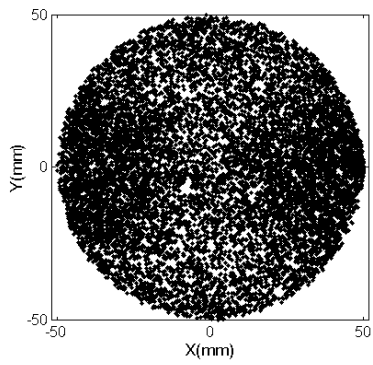

(a)

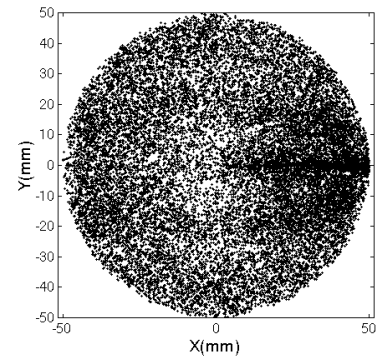

(b)
Figure 2 Photoelectron distributions in transverse section for $\mathrm{C}$ yoke dipole field

(a) equal polarity configuration;

(b) alternating polarity configuration

The C-Yoke magnet can be arranged as a quadrupole magnet with field

$$
\begin{aligned}
B_{x} & =(a+b \cos k z) y \\
B_{y} & =(a+b \cos k z) x \\
B_{z} & =-b k \sin (k z) x y
\end{aligned}
$$

where $\quad a=0.3 \mathrm{~T} / \mathrm{m}, \quad b=0.2 \mathrm{~T} / \mathrm{m}, \quad \lambda=0.1 \mathrm{~m} \quad$ and $\quad a=0$, $b=0.5 \mathrm{~T} / \mathrm{m}, \lambda=0.2 \mathrm{~m}$ for the equal polarity and alternating polarity, respectively. Figure 3 shows the distribution of photoelectrons in transverse section for these two fields. The average volume density are $1.27 \times 10^{11} \mathrm{~m}^{-3}$ and $1.78 \times 10^{11} \mathrm{~m}^{-3}$, respectively. The central volume density are $0.64 \times 10^{11} \mathrm{~m}^{-3}$ and $1.59 \times 10^{11}$ $\mathrm{m}^{-3}$, respectively. The decay time is almost the same in the two cases. The equal polarity configuration is better because the central density is lower.

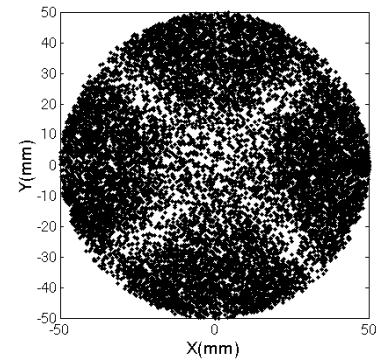

(a)

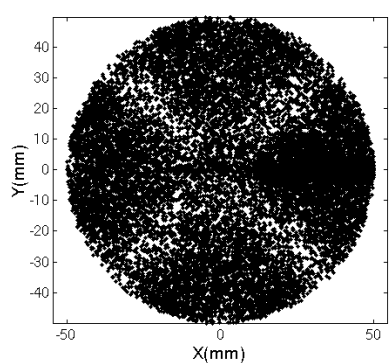

(b)
Figure 3 Photoelectron distribution in transverse section for $\mathrm{C}$ yoke quadrupole field

(a) equal polarity configuration

(b) alternating polarity configuration

\subsection{Uniform longitudinal field}

Figure 4 shows the distribution of electron cloud in transverse plane for uniform longitudinal magnetic field with strength 10 Gauss and 20 Gauss, respectively. The uniform solenoid field is very effective to confine the photoelectron. 20 Gauss uniform field is enough. Most of the photoelectrons are confined to the vicinity of the vacuum chamber wall. The average volume density is $2.99 \times 10^{11} \mathrm{~m}^{-3}$ and $1.08 \times 10^{11} \mathrm{~m}^{-3}$ for $B=10 \mathrm{G}$ and $B=20 \mathrm{G}$, respectively. The most important is that there is a large photoelectron free region at pipe center. The photoelectron takes about $10 \mathrm{~ns}$ to drift few $\mathrm{mm}$ in the vacuum chamber to reach the pipe wall under such magnet field.

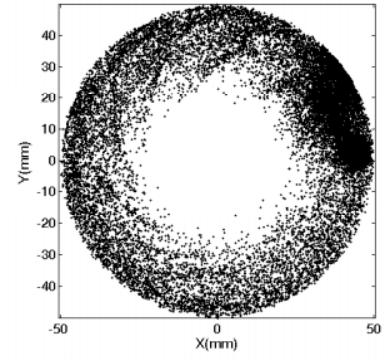

(a) $B=10 \mathrm{G}$

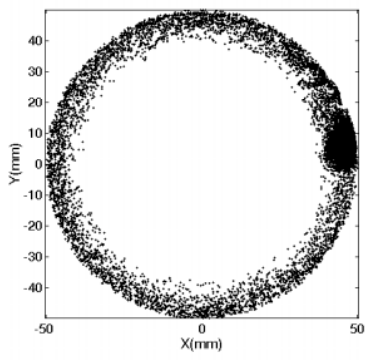

(b) $B=20 \mathrm{G}$
Figure 4 Photoelectron distributions in transverse section in uniform longitudinal magnetic field

\subsection{Solenoid field}

When the periodic solenoids are arranged with the same current direction in the coil, we call this kind of 
arrangement equal polarity configuration. In this case, the magnetic field can be approximately expressed as

$$
\begin{aligned}
& B_{z}(x, y, z)=B_{z 0}+B_{0} \sin k z, \\
& B_{x}(x, y, z)=-0.5 B_{0} k x \cos k z, \\
& B_{y}(x, y, z)=-0.5 B_{0} k y \cos k z .
\end{aligned}
$$

When the solenoids current takes alternating direction, which is called opposite polarity configuration, the longitudinal filed is expressed as

$$
B_{z}(x, y, z)=B_{0} \sin k z .
$$

The transverse field components are the same as equal polarity case. The simulated electron cloud distribution with $\mathrm{B}_{\mathrm{z} 0}=30$ Gauss, $\mathrm{B}_{0}=20$ Gauss, $\lambda=1 \mathrm{~m}$ and $\mathrm{B}_{0}=50$ Gauss, $\lambda=2 \mathrm{~m}$ for equal and opposite polarity case is shown in figure 5 . The volume densities in equal polarity and opposite polarity case are $2.21 \times 10^{11} \mathrm{~m}^{-3}$ and $2.61 \times 10^{11} \mathrm{~m}^{-1}$, respectively. The decay time in the train gap is $290 \mathrm{~ns}$ and $245 \mathrm{~ns}$, respectively. There is an electron free region and a low electron density of $0.25 \times 10^{11} \mathrm{~m}^{-3}$ region at the pipe center for the equal and opposite polarity case, respectively. Another paper[6] discusses the calculation in more details.

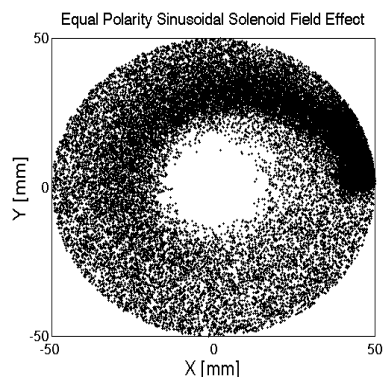

(a)

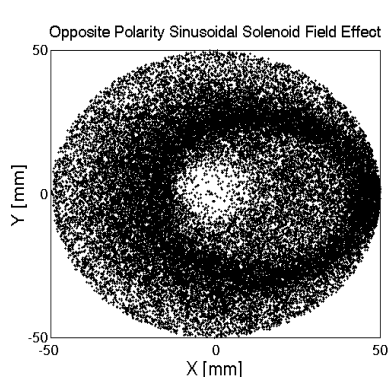

(b)
Figure 5 Photoelectron distributions in transverse section in periodic solenoid field (a: equal polarity configuration; $b$ : alternating polarity configuration)

\subsection{Normal dipole and quadrupole magnet}

Most of bending magnets in LER are normal bending magnets with $\mathrm{B}=0.848 \mathrm{~T}$. A gradient of $10.3 \mathrm{~T} / \mathrm{m}$ is assumed for the normal quadrupole in simulation. Figure 6 shows the photoelectron distribution in these two kinds of magnets. The e-cloud distribution in normal dipole magnet is different from that in the dipole $\mathrm{C}$-Yoke because the field pattern and strength is different. The average and central volume density in normal dipole magnet are $0.98 \times 10^{11} \mathrm{~m}^{-3}$ and $0.28 \times 10^{11}$ $\mathrm{m}^{-3}$. The pattern of the e-cloud in a normal quadrupole is quite similar with that in the C-Yoke quadrupole magnet. The average volume density is $1.78 \times 10^{11} \mathrm{~m}^{-3}$, which is same as C-Yoke alternating polarity case. However, the volume density at pipe center, $0.14 \times 10^{11}$ $\mathrm{m}^{-3}$, is few times lower than that of C-Yoke case. The density of photoelectron in various kinds of magnet fields is summarized in table 2 .

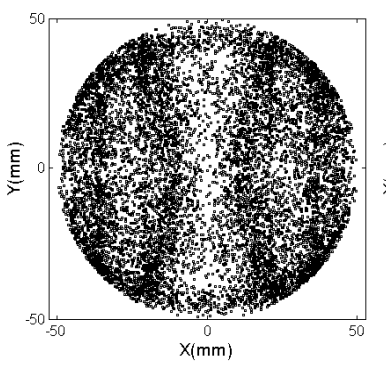

(a) Normal dipole

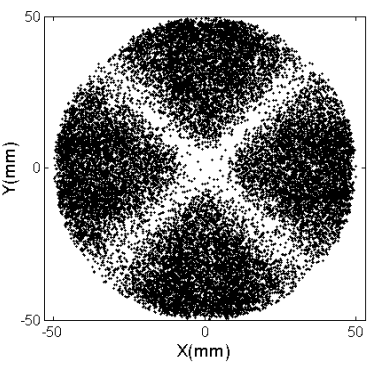

(b) Normal quadrupole
Figure 6 Photoelectron distributions in transverse section in normal dipole and quadrupole magnet

Table 2 Photoelectron density in various magnet

\begin{tabular}{|c|c|c|}
\hline Magnet Field & $\begin{array}{c}\rho_{\mathrm{a}} \\
\left(\times 10^{11} \mathrm{~m}^{-3}\right)\end{array}$ & $\begin{array}{c}\rho_{\mathrm{c}} \\
\left(\times 10^{11} \mathrm{~m}^{-3}\right)\end{array}$ \\
\hline Field Free & 2.78 & 8.83 \\
\hline C-Yoke Dipole (EP) & 1.07 & 0.75 \\
\hline C-Yoke Dipole (AP) & 0.98 & 0.35 \\
\hline C-Yoke Quadrupole (EP) & 1.27 & 0.64 \\
\hline C-Yoke Quadrupole(AP) & 1.78 & 1.59 \\
\hline Uniform B=10G & 2.99 & $<2.5 \times 10^{-4}$ \\
\hline Uniform B=20G & 1.08 & $<2.5 \times 10^{-4}$ \\
\hline Solenoid (EP) & 2.21 & $<2.5 \times 10^{-4}$ \\
\hline Solenoid(AP) & 2.61 & 0.25 \\
\hline Normal Dipole & 0.98 & 0.28 \\
\hline Normal Quadrupole & 1.78 & 0.14 \\
\hline
\end{tabular}

Symbol in table: $\boldsymbol{\rho}_{\mathbf{a}}$ : Average volume density; $\boldsymbol{\rho}_{\mathbf{c}}$ : Centra volume density, EP: equal polarity, AP: alternating polarity.

\section{SUMMARY AND CONCLUSIONS}

The simulation shows that the magnetic field can reduce the electron density at the pipe center. However, it has little effect on reducing the electron average density. Uniform solenoid field is the most effective field to confine the photoelectron to the vicinity of the vacuum chamber wall and solenoid is better than C-Yoke magnet.

\section{REFERENCES}

[1] H. Fukuma, et al., "Observation of vertical beam blow-up in KEKB low energy ring", EPAC2000, 2000.

[2] F.Zimmermann, "Electron-Cloud Studies for the LowEnergy Ring of KEKB", CERN-SL-Note-004(AP), 2000.

[3] K. Ohmi and F. Zimmermann, "Head-tail instability caused by electron cloud n positron storage rings", Physics Review Letter (85):3821, 2000.

[4] H. Fukuma et al., "Study of vertical beam blowup in KEKB low energy ring", HEACC2001.

[5] L.F. Wang, H. Fukuma, K. Ohmi, "3D PIC Space Charge Solver Based on P3M method", to be published in APAC2001.

[6] L.F. Wang, H. Fukuma, K. Ohmi, "Simulation Study of Photoelectron Motion in Solenoid Field", KEK-2001-2, 2001. 